Pacific Journal of Mathematics

THE ABSOLUTE INVARIANCE OF CONSERVATION LAWS 


\title{
THE ABSOLUTE INVARIANCE OF CONSERVATION LAWS
}

\section{H. H. JoHNSON}

\begin{abstract}
The only known absolute invariant for a general system of pde is its maximal character. The purpose of this paper is to prove that conservation laws are also absolute invariants, that is, they are preserved under partial prolongations in a natural way. We also show that the property, closely related to soliton behavior, of having an infinite number of conservation laws is an absolute invariant.
\end{abstract}

\section{Introduction and background. Partial prolongations of systems of} pde were introduced by E. Cartan to define when two continuous infinite dimensional transformation pseudogroups are equivalent as abstract groups $[1$, p. $625 \mathrm{ff}]$. With this relation he was able to classify the simple infinite pseudogroups $[1$, p. $857 \mathrm{ff}]$.

In $[1$, p. 1133] Cartan studied partial prolongations of general systems of pde and used them to make precise the notion due to $D$. Hilbert of when two systems possess a one-to-one correspondence between their solutions [6]. Two systems are "absolutely equivalent" when they can be joined by a finite sequence of systems where for each adjacent pair in the sequence, one is a partial prolongation of the other.

Thus, the main use of partial prolongations is geometric: a property associated with systems of pde is an "absolute invariant" property when it is shared by both systems in any partial prolongation.

In a series of papers the author showed that several classical concepts (hyperbolicity, characteristics) are not, in fact, absoluted invariants $[7,8,9,10,11]$. This means that these concepts are not necessarily intrinsically related to the systems they are defined on: if one changes the form of the system by a partial prolongation, such concepts may change or no longer exist. Any theory based on these non-absolute invariants may predict different kinds of behavior for systems which are in fact completely and naturally equivalent.

In physics the problem is even more crucial. Concepts which are not absolute invariants of the systems of pde that describe the physical 
process are not "physical" at all, since they could change or disappear if one alters the describing system of pde in some inessential way: they depend on how you happen to write down the pde. Just as modern physics commonly seeks quantities which are shared by systems equivalent under geometric Lie groups, they ought to also check for invariance under partial prolongations.

A rare concept which does turn out to be an absolute invariant would thus be particularly important and the focus of special interest. Until the present paper, the only known absolute invariant is the maximal character, which determines, roughly, the number of arbitrary functions in a maximal number of independent variables which can be assigned arbitrarily in the initial value problem [10, p. 237]. In this paper we prove conservations laws to be absolutely invariant.

In some works, pde which are derived from a conservation law are studied [12]. Our viewpoint is the opposite: to study the conservation laws associated with a given system of pde. Studies of the KdV equation and others reveal that they have conservation laws related to soliton solutions.

Solitons are special solutions associated with certain pde [2]. Since we shall show that there is a one-to-one correspondence between the solutions of absolutely equivalent systems, the property of having soliton solutions is an absolute invariant. The property of having infinitely many different conservation laws seems closely connected with existence of solitons [2, p. 36]. We show that this property is also an absolute invariant, thus strengthening this conjectured connection.

To make this paper as readable and self-contained as possible, we illustrate the ideas with numerous examples and often use local coordinates. Experts can easily translate the proofs into the language of abstract fiber spaces and bundles.

We do take for granted a familiarity with the calculus of exterior differential forms, however [4].

\section{Examples of conservation laws.}

EXAMPLE $2.1[2$, p. 32]. Let a compressible fluid of density $r(x, t)$ be flowing with velocity $u(x, t)$ in the $x$-direction at time $t$. Then

$$
\frac{\partial r}{\partial t}+\frac{\partial(r u)}{\partial x}=0
$$


Consider the 1 -form $C=r d x-(r u) d t$. If we compute $d C$, the exterior derivative of $C$, using exterior algebra and calculus, we get

$$
\begin{aligned}
d C & =\left(\frac{\partial r}{\partial x} d x+\frac{\partial r}{\partial t} d t\right) \wedge d x-\left(\frac{\partial(r u)}{\partial x} d x+\frac{\partial(r u)}{\partial t} d t\right) \wedge d t \\
& =\left(\frac{\partial r}{\partial t}+\frac{\partial(r u)}{\partial x}\right) d t \wedge d x=0,
\end{aligned}
$$

when one "takes into account" equation (2.1).

When $E$ is a plane region in $(x, t)$-space with oriented boundary $\partial E$

$$
\oint_{\partial E} C=\iint_{E} d C=0 .
$$

If $E=\left\{(x, t) \mid-\infty<x<\infty, t_{1} \leq t \leq t_{2}\right\}$ then the last equation says the total mass at time $t_{1}$ equals the total mass at time $t_{2}$.

On the other hand, when $E=\left\{(x, t) \mid x_{1} \leq x \leq x_{2},-\infty<t<\infty\right\}$, it expresses conservation of total flux across two planes at $x_{1}$ and $x_{2}$.

The 3-dimensional analogue works the same way, only now $C$ is a 3-form.

EXAMPLE 2.2. The plane motion of $N$ particles of masses $m_{i}$ at $\left(x^{i}, y^{i}\right)$ under gravity is governed by

$$
\begin{aligned}
& m_{i} \frac{d^{2} x^{i}}{d t^{2}}=k \sum_{j \neq i} \frac{x^{j}-x^{i}}{d_{i j}} m_{i} m_{j}, \\
& m_{i} \frac{d^{2} y^{i}}{d t^{2}}=k \sum_{j \neq i} \frac{y^{j}-y^{i}}{d_{i j}} m_{i} m_{j},
\end{aligned}
$$

where

$$
d_{i j}=\left[\left(x^{i}-x^{j}\right)^{2}+\left(y^{i}-y^{j}\right)^{2}\right]^{3 / 2}, \quad i, j=1, \ldots, N .
$$

Let

$$
L=\sum_{i=1}^{N} m_{i}\left(x^{i} \frac{d y^{i}}{d t}-y^{i} \frac{d x^{i}}{d t}\right)
$$

If we replace

$$
d x^{i} \quad \text { by } \frac{d x^{i}}{d t} d t, \quad d\left(\frac{d x^{i}}{d t}\right) \text { by } \frac{d^{2} x^{i}}{d t^{2}} d t, \quad \text { etc. }
$$

then $d L=0$ when we "take into account" equations (2.2).

Notice that we must treat $d x^{i} / d t$ and $d y^{i} / d t$ as variables in some extended space. Ehresmann's "jet" manifolds are just such a space [3] where, indeed, they are coordinates. 
EXAMPLE 2.3 (KdV Equation).

$$
\frac{\partial y}{\partial t}-6 y \frac{\partial y}{\partial x}+\frac{\partial^{3} y}{\partial x^{3}}=0 .
$$

This is known to have soliton solutions [2, pp. 3-8]. Then, if

$$
\begin{aligned}
C= & y d x-\left(\frac{\partial^{2} y}{\partial x^{2}}-3 y^{2}\right) d t, \\
d C= & d y \wedge d x-\left(d\left(\frac{\partial^{2} y}{\partial x^{2}}\right)-6 y d y\right) \wedge d t \\
= & \left(\frac{\partial y}{\partial x} d x+\frac{\partial y}{\partial t} d t\right) \wedge d x \\
& -\left[\frac{\partial^{3} y}{\partial x^{3}} d x+\frac{\partial^{3} y}{\partial x^{2} \partial t} d t-6 y\left(\frac{\partial y}{\partial x} d x+\frac{\partial y}{\partial t} d t\right)\right] \wedge d t \\
= & \left(\frac{\partial y}{\partial t}+\frac{\partial^{3} y}{\partial x^{3}}-6 y \frac{\partial y}{\partial x}\right) d t \wedge d x=0,
\end{aligned}
$$

if one "takes into account" (2.3).

EXAMPLE 2.4. Consider next the "formal derivative" of $C$ in the last example:

$$
\partial_{x} C=\frac{\partial y}{\partial x} d x-\left(\frac{\partial^{3} y}{\partial x^{3}}-6 y \frac{\partial y}{\partial x}\right) d t .
$$

By using not only equation (2.3) but the derivative of equation (2.3) with respect to $x$, one finds that $d\left(\partial_{x} C\right)=0$ if one replaces

$$
d\left(\frac{\partial y}{\partial x}\right) \text { by } \frac{\partial^{2} y}{\partial x^{2}} d x+\frac{\partial^{2} y}{\partial x \partial t} d t, \text { etc. }
$$

We thus find that the formal derivative of the conservation law $C$ is also a conservation law. This is true in general. The $\mathrm{KdV}$ equation is unusual in that it has an infinite number of conservation laws which are not formal derivatives of some lower order laws [2, pp. 34-36].

EXAMPLE 2.5.

$$
\frac{\partial^{2} y}{\partial x^{1} \partial x^{2}}=0, \quad C=\left(\frac{\partial y}{\partial x^{1}}\right)^{2} d x^{1}+\frac{\partial y}{\partial x^{2}} d x^{2} .
$$

Then

$$
d C=\left[2\left(\frac{\partial y}{\partial x^{1}}\right)\left(\frac{\partial^{2} y}{\partial x^{1} \partial x^{2}}\right)-\frac{\partial^{2} y}{\partial x^{1} \partial x^{2}}\right] d x^{2} \wedge d x^{1}=0
$$

when one uses equation (2.5). 
We observe that in each case an exterior form $C$ over the differentials of the independent variables is given, whose coefficients are functions of the dependent and independent variables and certain partial derivatives. Moreover, in computing the exterior derivative of $C$ one must use certain 1-forms to obtain $d C$ in terms of the differentials of the independent variables. Then, when the original equation or some of its formal derivatives are "taken into account," $d C=0$.

We want first to make this process precise and reduced to ordinary computations on the geometry of manifolds. This can be done using Ehresmann's jet spaces.

II. Jet spaces. C. Ehresmann's theory of jets [3] enables us to solve problems about the structure of pde by differential geometric methods [13]. We introduce notations and state elementary results that will be needed.

If $X=\mathbb{R}^{n}$ has coordinates $\left(x^{1}, \ldots, x^{n}\right)$ and $Y=\mathbb{R}^{m}$ has coordinates $\left(y^{1}, \ldots, y^{m}\right)$ then the space $J_{p}$ of $p$-jets of maps from $X$ to $Y$ has coordinates that correspond to the variables in a pde of order $p$ with dependent variables $y^{j}$ and independent variables $x^{i}$, namely,

$$
x^{i}, y^{j}, y_{k}^{j}, y_{h j}^{j}, \ldots \text { up through order } p
$$

(where $y_{h j}^{j}$ represents $\partial y^{j} / \partial x^{h} \partial x^{k}$, etc.)

Ehresmann gave the elegant definition: a $p$-jet at $x \in X$ is the equivalence class $j_{p}(f)(x)$ of a local $C^{\infty}$ function $f: X \rightarrow Y$ whose domain includes $x$, the equivalence relation being:

$f \approx g$ if $f(x)=g(x)$ and all partials of $f$ and $g$ are the same at $x$ up to and including order $p$. Then in coordinates the jet $j_{p}(f)(x)$ is

$$
\left(x^{i}, f^{j}(x), \frac{\partial f^{j}}{\partial x^{k}}(x), \frac{\partial^{2} f}{\partial x^{h} \partial x^{k}}(x), \ldots\right) \text {. }
$$

The fact that $y_{l k}^{j}=y_{k i}^{j}$ complicates things. A very convenient notation from pde theory is

$$
y_{i_{1} i_{2} \cdots i_{n}}^{j}=\frac{\partial^{r} f^{j}}{\partial\left(x^{1}\right)^{i_{1}} \partial\left(x^{2}\right)^{i_{2}} \cdots \partial\left(x^{n}\right)^{i_{n}}}=\partial_{i_{1} \cdots i_{n}} f^{j}
$$

rather than $y_{11 \cdots 22 \cdots n}$. For this " $n$-tuple" notation we designate $\nu=$ $\left(i_{1}, \ldots, i_{n}\right)$ where $i_{k}$ are non-negative integers, so

$$
y_{i_{1}, \ldots, i_{n}}^{j}=y_{\nu}^{j}=\partial_{\nu} f^{j} .
$$

Let $|\nu|=i_{1}+\cdots+i_{n}$. If we let $y_{0 \cdots 0}^{j}=y^{j}$, then the coordinates of $J_{p}$ are $\left(x^{l}, y_{\nu}^{j}\right)$, for $|\nu| \leq p$. 
It is understood that the $i$ and $j$ depend on dimensions of $X$ and $Y$. We often omit these upper indices $i, j$ which greatly simplifies the notation, and merely write $\left(x, y_{\nu}\right),|\nu| \leq p$ for coordinates in $J_{p}[\mathbf{1 4}$, 15].

We also simplify things by using the summation convention.

The reason why it is necessary to involve the geometry of $J_{p}$ is that we want results independent of choice of coordinates on $X$ or $Y$. If we allow changes of variables of the form $y^{\prime}=F(x, y), x^{\prime}=G(x)$, we are really studying jets $X \rightarrow E$, where $E$ is a fibered manifold $\pi: E \rightarrow X$. That is the viewpoint in $[14,15]$.

(The physicist may need even more general coordinate changes $y^{\prime}=$ $F(x, y), x^{\prime}=G(x, y)$. The author knows of no extension of jet formalism to this situation. Cartan's theory of exterior systems of equations is, however, of this genre, since it makes no implicit designation of independent variables.)

Notation. If $\nu=\left(i_{1}, \ldots, i_{n}\right)$, then

$$
\nu+1_{j}=\left(i_{1}, \ldots, i_{j}+1, \ldots, i_{n}\right) .
$$

If $f: X \rightarrow Y$ is a local $C^{\infty}$ function then $x \rightarrow j_{p}(f)(x)$ defines a map from $X$ to $J_{p}$, given by

$$
j_{\nu}(f)=\left\{\begin{array}{l}
x=x \\
y_{\nu}=\partial_{\nu} f, \nu=\left(i_{1}, \ldots, i_{n}\right) .
\end{array}\right.
$$

For such an $f$,

$$
d y_{\nu}=\sum_{i=1}^{n} \partial_{\nu+1} f d x^{i}=\sum y_{\nu+1} d x^{i}, \quad|\nu|<p .
$$

Hence, the "pull-backs" by $j_{p}(f)$ of the 1 -forms

$$
w_{\nu}=d y_{\nu}-\sum y_{\nu+1} d x^{i}, \quad|\nu|<p,
$$

are all zero: $j_{p}(f)^{*}\left(w_{\nu}\right)=0$.

Definition 3.1. The 1-forms $w_{\nu}^{j}=d y_{\nu}^{j}-y_{v+1}^{j} d x^{i}$, are the contact 1 -forms of $J_{p}$. They generate the contact ideal $\Omega_{p}$ on $J_{p}$. It is known that $g: X \rightarrow J_{p}$ equals $j_{p}(f)$ for some $f: X \rightarrow Y$ if and only if $g^{*}\left(\Omega_{p}\right)=0$ [13, p. 20ff].

Now we consider real-valued functions $\Phi: J_{p} \rightarrow \mathbb{R}$.

Definition 3.2. The formal (partial) derivative of $\Phi$ with respect to $x^{i}$ is $\partial_{i} \Phi\left(j_{p+1}(f(x))\right)=\left[\frac{\partial\left[\Phi \circ j_{p}(f)\right]}{\partial x^{i}}\right](x)$. This is well-defined, and 
in coordinates looks like

$$
\partial_{i} \Phi=\frac{\partial \Phi}{\partial x^{i}}+\sum_{|\nu| \leq p} \frac{\partial \Phi}{\partial y_{\nu}} y_{\nu+1} .
$$

EXAMPle 3.1. Let $p=2, \operatorname{dim} X=2, \operatorname{dim} Y=1$. Let

$$
\Phi\left(x^{1}, x^{2}, y, y_{10}, y_{01}, y_{20}, y_{11}, y_{02}\right)=x^{1} y_{01}+y_{20} y_{11} \text {. }
$$

Then $\partial_{1} \Phi=y_{01}+x^{1} y_{11}+y_{30} y_{11}+y_{30} y_{11}+y_{20} y_{21}$.

We could also use the $n$-tuple notation $\partial_{10} \Phi$ instead of $\partial_{1} \Phi$. More generally, for any $n$-tuple $\mu, \partial_{\mu} \Phi: J_{p+|\mu|} \rightarrow \mathbb{R}$. is defined, and

Proposition 3.1. $\partial_{\nu}\left(\partial_{\mu} \Phi\right)=\partial_{\mu}\left(\partial_{\nu} \Phi\right)=\partial_{\nu+\mu} \Phi$.

Instead of studying systems of equations on $J_{p}$ we study their zero sets, which we assume to be submanifolds of $J_{p}$.

Definition 3.3. A system of pde of order $p$ is a submanifold $R_{p}$ of $J_{p}$. A local map $f: X \rightarrow Y$ is a solution of $R_{p}$ when, for all $x \in \operatorname{domain}(f), j_{p}(f)(x) \in R_{p}$.

The "prolongation" $R_{p+1}$ of $R_{p}$ is obtained by formally differentiating the equations that define $R_{p}$. For example, (2.4) would be one of the equations defining the prolongation of the $\mathrm{KdV}$ equation.

Definition 3.4. Let $R_{p}=\left\{\left(x, y_{\lambda}\right) \mid g\left(x, y_{\lambda}\right)=0\right\}$. Then

$$
R_{p+1}=\left\{\left(x, y_{\mu}\right) \in J_{p+1} \mid g\left(x, y_{\lambda}\right)=0, \partial_{j} g\left(x, y_{\mu}\right)=0,1 \leq j \leq n\right\}
$$

is the first prolongation of $R_{p}$. More generally

$$
R_{p+q}=\left\{\left(x, y_{\mu}\right) \in J_{p+q}\left|\partial_{\nu} g\left(x, y_{\mu}\right)=0,0 \leq\right| \nu \mid \leq q\right\} .
$$

Let $\Pi_{p}^{p+q}: J_{p+q} \rightarrow J_{p}$ be the projection. Then obviously

Proposition 3.2. $\Pi_{p}^{p+q}\left(R_{p+q}\right) \subseteq R_{p}$.

Moreover, it can be shown that

Proposition 3.3. If $F: J_{p} \rightarrow \mathbb{R}$ is zero on $R_{p}$, then $\partial_{\nu} F: J_{p+q} \rightarrow \mathbb{R}$ is zero on $R_{p+q}$, for all $|\nu| \leq q$. (Note that though $R_{p}$ is assumed to be a manifold, $R_{p+q}$ need not be.)

More generally, suppose $Y^{\prime}$ is another manifold and $J_{r}^{\prime}$ is the manifold of $r$-jets of maps $f^{\prime}: X \rightarrow Y^{\prime}$. We need to consider maps 
$F: J_{p} \rightarrow X \times Y^{\prime}$ which preserve $X$, i.e., $F\left(j_{p}(f)(x)\right)=\left(x, y^{\prime}\right)$ (same point $x$ ). In coordinates:

$$
F:\left\{\begin{array}{l}
x=x, \\
y^{\prime}=\varphi\left(x, y_{\lambda}\right), \quad|\lambda| \leq p .
\end{array}\right.
$$

Definition 3.5. Let $F: J_{p} \rightarrow X \times Y^{\prime}$ preserve $X$. In this case the prolongation of $F, \rho_{q}(F): J_{p+q} \rightarrow J_{q}^{\prime}$, is defined by

$$
\rho_{q}(F):\left\{\begin{array}{l}
x=x, \\
y_{\mu}^{\prime}=\partial_{\mu} \Phi\left(x, y_{\theta}\right), \quad|\theta| \leq p+q,|\mu| \leq q .
\end{array}\right.
$$

(For invariant definitions of 3.4 and 3.5 see $[14$, pp. 40, 49].)

Proposition 3.4. Let $F: J_{p} \rightarrow X \times Y^{\prime}$ preserve $X$. Suppose $R_{p+q} \subseteq$ $J_{p+q}$ and $R_{q}^{\prime} \subseteq J_{q}^{\prime}$ are defined as the zero sets of some functions. If $\rho_{q}(F)\left(R_{p+q}\right) \subseteq R_{q}^{\prime}$, then, for all $r \geq 0$,

$$
\rho_{q+r}(F)\left(R_{p+q+r}\right) \subseteq R_{q+r}^{\prime}
$$

IV. Partial prolongations. To explain the idea of a partial prolongation consider the following examples.

EXAMPLE 4.1. Let $\operatorname{dim} X=2, \operatorname{dim} Y=1, R_{2} \subset J_{2}$ defined by

$$
\frac{\partial^{2} y}{\partial x^{1} \partial x^{2}}=y_{11}=0 \text {. }
$$

Let $R_{1}^{\prime}$ be the system on $X \times Y \times Z, \operatorname{dim} Z=2$, defined by

$$
R_{1}^{\prime}: z^{1}=y_{10}, \quad z^{2}=y_{01}, \quad z_{01}^{1}=z_{10}^{2}=0 .
$$

Obviously, $R_{2}$ and $R_{1}^{\prime}$ are related. The new variables $z^{1}, z^{2}$ are the first derivatives of the variable $y$ in $R_{2}$. The last equations of $R_{1}^{\prime}$ say the same thing as the equation $y_{11}=0 . R_{1}^{\prime}$ is called a partial prolongation of $R_{2}$.

(This device of introducing new variables for lower order derivatives of variables in a pde can be used to reduce any pde to a first order system, called its "lowered system" [14, p. 109ff].)

EXAMPLE 4.2.

$$
\begin{aligned}
& R_{2}: y_{11}=0, \\
& R_{1}^{\prime}: y_{01}=y-A z, \quad y_{10}-A z_{10}=0 \quad(A=\text { constant }) .
\end{aligned}
$$

Suppose $y\left(x^{1}, x^{2}\right), z\left(x^{1}, x^{2}\right)$ solves $R_{1}^{\prime}$. Differentiating the first equation that defines $R_{1}^{\prime}$ gives $y_{11}=y_{10}-A z_{10}=0$, so $y$ solves $R_{2}$. 
On the other hand, when $y$ solves $R_{2}$, if $z=\left(y-y_{01}\right) / A$ then $y_{10}-A z_{10}=0$, so $(y, z)$ solves $R_{1}^{\prime}$. There is thus a natural one-toone correspondence between the solutions of the two systems. $R_{1}^{\prime}$ is a "partial prolongation" of $R_{2}$.

\section{EXAMPLE 4.3.}

$$
\begin{array}{lll}
R_{1}: y_{01}^{1}=0, & y_{01}^{2}=y_{10}^{2}, & y_{01}^{3}=y_{10}^{1}, \\
R_{1}^{\prime}: y_{01}^{1}=0, & y_{01}^{2}=y_{10}^{2}, & y_{01}^{3}=y_{10}^{1}, \quad z=y_{10}^{1}, \quad z_{01}=0 .
\end{array}
$$

Again, there is a one-to-one natural correspondence between the solutions of $R_{1}$ and $R_{1}^{\prime}$.

DEFINITION 4.1. Let $R_{p} \subset J_{p}$ be a system with independent variable manifold $X$ and dependent variable manifold $Y$. Let $Z$ be a manifold and

$$
F: J_{p-r} \rightarrow X \times Y \times Z \quad \text { for some } 0<r \leq p-1 .
$$

Let $J_{r}^{\prime}$ be the $r$-jets of maps $X \rightarrow Y \times Z$. Denote by $\Pi_{y}: X \times Y \times Z \rightarrow$ $X \times Y$ the projection.

Let $R_{r}^{\prime} \subset J_{r}^{\prime}$ be a system of pde, $R_{p}^{\prime}$ its $(p-r)$ th projection. Assume

(1) $\Pi_{y} \circ F=\Pi_{0}^{p-r}: J_{p-r} \rightarrow X \times Y$,

(2) $\rho_{r}(F): J_{p} \rightarrow J_{r}^{\prime}$ satisfies $\rho_{r}(F)\left(R_{p}\right) \subset R_{r}^{\prime}$,

(3) $\rho_{p}\left(\Pi_{y}\right): J_{p}^{\prime} \rightarrow J_{p}$ satisfies $\rho_{p}\left(\Pi_{y}\right)\left(R_{p}^{\prime}\right) \subset R_{p}$, and

(4) $F \circ \Pi_{p-r}^{p} \circ \rho_{p}\left(\Pi_{y}\right): J_{p}^{\prime} \rightarrow X \times Y \times Z$ satisfies $F \circ \Pi_{p-r}^{p} \circ \rho_{p}\left(\Pi_{y}\right)=\Pi_{0}^{\prime p}$ on $R_{p}^{\prime}$.

Then we call $R_{r}^{\prime}$ a partial prolongation of $R_{p}$

\section{Commutative Diagram}

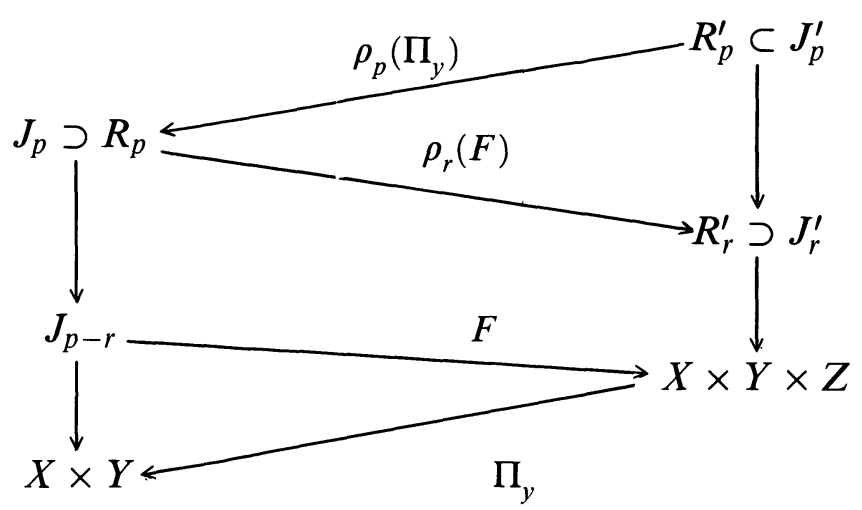

COORDINATES 
Choose $(x)$ on $X,(y)$ on $Y$ and $(z)$ on $Z$. Then condition (1) implies

$$
F:\left\{\begin{array}{l}
x=x, \\
y=y, \\
z=\varphi\left(x, y_{\theta}\right),|\theta| \leq p-r
\end{array} \quad \Pi_{y}:\left\{\begin{array}{l}
x=x \\
y=y
\end{array}\right.\right.
$$

$$
\rho_{r}(F): \begin{cases}x=x, & \\ y_{\nu}=y_{\nu}, & |\nu| \leq r, \\ z_{\nu}=\left(\partial_{\nu} \varphi\right)\left(x, y_{\lambda}\right), & |\lambda| \leq r+p-r=p .\end{cases}
$$

$$
\rho_{p}\left(\Pi_{y}\right):\left\{\begin{array}{l}
x=x, \\
y_{\lambda}=y_{\lambda}
\end{array} \quad|\lambda| \leq p .\right.
$$

Condition (2) says

$$
\text { if }\left(x, y_{\lambda}\right) \in R_{p} \quad \text { then }\left(x, y_{\nu},\left(\partial_{\nu} \varphi\right)\left(x, y_{\lambda}\right)\right) \in R_{r}^{\prime} .
$$

Condition (3) says

$$
\text { if }\left(x, y_{\lambda}, z_{\lambda}\right) \in R_{p}^{\prime} \quad \text { then }\left(x, y_{\lambda}\right) \in R_{p} .
$$

\section{Condition (4) says}

(4.6) if $\left(x, y_{\lambda}, z_{\lambda}\right) \in R_{p}^{\prime} \quad$ then $z=\varphi\left(x, y_{\theta}\right)$ for $|\theta| \leq p-r$.

That is, $z-\varphi\left(x, y_{\theta}\right)=0$ on $R_{p}^{\prime}$.

EXAMPLE 4.1. (See above.)

$$
\rho_{1}(F):\left\{\begin{array}{l}
x=x \\
y=y \\
z^{1}=y_{10}=\varphi^{1}\left(x, y_{\nu}\right) \\
z^{2}=y_{01}=\varphi^{2}\left(x, y_{\nu}\right) \\
y_{\nu}=y_{\nu}, \quad|\nu|=1 \\
z_{10}^{1}=y_{20}, \\
z_{01}^{1}=y_{11}, \\
z_{10}^{2}=y_{11}, \\
z_{01}^{2}=y_{02}
\end{array}\right.
$$

Condition (2): if $\left(x, y_{\lambda}\right) \in R_{2}, y_{11}=0$, so $z_{01}^{1}=z_{10}^{2}=0$, and hence $\left(x, y_{\nu}, z_{\nu}\right)=\rho_{1}(F)\left(x, y_{\lambda}\right) \in R_{1}^{\prime}$. 
Condition (3): $R_{2}^{\prime}$ is defined by the equations defining $R_{1}^{\prime}$ together with $z_{10}^{1}=y_{20}, 0=z_{01}^{1}=y_{11}=z_{10}^{2}, y_{02}=z_{01}^{2}, z_{11}^{1}=z_{20}^{2}=$ $z_{02}^{1}=z_{11}^{2}=0$. If $\left(x, y_{\lambda}, z_{\lambda}\right) \in R_{2}^{\prime}$, then $y_{11}$ must be zero; hence $\left(x, y_{\lambda}\right) \in R_{2}$.

Condition (4): if $\left(x, y_{\lambda}, z_{\lambda}\right) \in R_{2}^{\prime}$ then $z^{1}=y_{10}=\varphi^{1}\left(x, y_{\theta}\right)$ and $z^{2}=y_{01}=\varphi^{2}\left(x, y_{\theta}\right)$, since these equations are among the defining equations of $R_{2}^{\prime}$.

EXAMPLE 4.2. This is equally easy to check, using

$$
\rho_{1}(F):\left\{\begin{array}{l}
F: x=x, y=y, z=\frac{y-y_{01}}{A}=\varphi\left(x, y_{0}\right), \\
y_{\nu}=y_{\nu}, z_{10}=\frac{y_{10}-y_{11}}{A}, z_{01}=\frac{y_{01}-y_{02}}{A} .
\end{array}\right.
$$

EXAmple 4.3. Here, $r=1, p-r=1$, so $p$ should be 2 . To fit the definition we must first prolong $R_{1}$ to $R_{2}$. Then $R_{1}^{\prime}$ can be shown to be a partial prolongation of $R_{2}$.

We next show how the solutions of a system and a partial prolongation correspond in a natural one-to-one way.

Let $R_{p}$ and $R_{r}^{\prime}$ be as in Definition 4.1.

THEOREM 4.1. For every local solution $f: X \rightarrow Y$ of $R_{p}$,

$$
P(f)=\prod_{y z} \circ F \circ j_{p-r}(f): X \rightarrow J_{p-r} \rightarrow X \times Y \times Z \rightarrow Y \times Z
$$

is a solution of $R^{\prime}$.

For every local solution $f^{\prime}: X \rightarrow Y \times Z$ of $R_{r}^{\prime}$,

$$
Q\left(f^{\prime}\right)=\widetilde{\Pi}_{y} \circ f^{\prime}: X \rightarrow Y \times Z \rightarrow Y
$$

is a solution of $R_{p}$.

Furthermore, $P \circ Q\left(f^{\prime}\right)=f^{\prime}$ and $Q \circ P(f)=f$.

Proof. Most of the complexity in this proof is notational, arising out of the usual confusion between functions and their graphs. In the local coordinates that follow Definition 4.1 it is quite transparent.

$$
\begin{aligned}
& F: x=x, \quad y=y, \quad z=\varphi\left(x, y_{\theta}\right), \quad|\theta| \leq p-r, \\
& f: y=f(x) \text { hence, }
\end{aligned}
$$

$$
P(f):\left\{\begin{array}{l}
y=f(x) \\
z=\varphi\left(x,\left(j_{\theta} f\right)(x)\right)
\end{array}\right.
$$


so by Definition 3.5,

$$
j_{r}(P(f)):\left\{\begin{array}{l}
x=x \\
y_{\nu}=\left(j_{\nu} f\right)(x), \\
z_{\nu}=\left(\partial_{\nu} \varphi\right)\left(x,\left(j_{\lambda} f\right)(x)\right) .
\end{array}\right.
$$

Since $f$ solves $R_{p},\left(x,\left(j_{\lambda} f\right)(x)\right) \in R_{p}$ for all $x \in \operatorname{domain}(f)$, and by (4.4) $\left(x, \partial_{\nu} f(x),\left(\partial_{\nu} \varphi\right)\left(x,\left(j_{\lambda} f\right)(x)\right)\right) \in R_{r}^{\prime}$, so $P(f)$ solves $R_{r}^{\prime}$. Let

$$
f^{\prime}:\left\{\begin{array}{l}
y=h(x), \\
z=k(x),
\end{array} \text { so } Q\left(f^{\prime}\right): y=h(x) .\right.
$$

Since $f^{\prime}$ solves $R_{r}^{\prime}$, it also solves $R_{p}^{\prime}$, and hence

$$
\left(x,\left(j_{\lambda} h\right)(x),\left(j_{\lambda} k\right)(x)\right) \in R_{p}^{\prime} \text { for }|\lambda| \leq p
$$

for all $x \in \operatorname{domain}\left(f^{\prime}\right)$. By (4.5), $\left(x,\left(j_{\lambda} h\right)(x)\right) \in R_{p}$ and hence $Q\left(f^{\prime}\right)$ solves $R_{p}$.

By (4.6), when $\left(x, y_{\lambda}, z_{\lambda}\right)=\left(x,\left(j_{\lambda} h\right)(x),\left(j_{\lambda} k\right)(x)\right) \in R_{p}^{\prime}, z=$ $k(x)=\varphi\left(x,\left(j_{\theta} h\right)(x)\right)$. Hence, $P \circ Q\left(f^{\prime}\right)=f^{\prime}$.

By (4.7) and (4.8), $Q \circ P(f)=f$.

\section{Conservation laws.}

Definition 5.1. Let $R_{p} \subset J_{p}$ be a system of pde. Let $C$ be an exterior differential $k$-form on $J_{s}$ which depends only on the differentials of the independent variables $\left(d x^{1}, \ldots, d x^{n}\right)$. Let $q>s$, and consider $d\left[\left(\Pi_{s}^{q}\right)^{*}(C)\right]$. Modulo the contact forms on $J_{q}$, this exterior derivative is congruent to a unique $(k+1)$-form in the differentials of the independent variables. We denote it by $d C / \Omega_{q}$.

Then $C$ is defined to be a conservation law for $R_{p}$ when, for some $q$ where $q \geq p$ and $q>s$,

$$
\text { if }\left(x, y_{\lambda}\right) \in R_{q} \quad \text { then } d C / \Omega_{q}\left(x, y_{\lambda}\right)=0 .
$$

(This is a slightly more general definition than given in [5, p. 253].)

\section{LOCAL COORDINATES}

Let

$$
C=\sum_{I}^{\prime} A_{I} d X^{I}, \quad A_{I}\left(x, y_{\mu}\right): J_{s} \rightarrow \mathbb{R}, \quad|\mu| \leq s .
$$

Here, $I=\left(i_{1}, i_{2}, \ldots, i_{k}\right), 1 \leq i_{1} \leq i_{2} \leq \cdots \leq n=\operatorname{dim} X$, and the $'$ means summation over all such ordered $k$-tuples, and $d X^{I}$ means 
$d x^{i} \wedge \cdots \wedge d x^{i}$. Then

$$
\begin{aligned}
& \left(\Pi_{S}^{q}\right)^{*}(C)=\sum_{I}^{\prime} A_{I} d X^{I} \quad \text { (same expression) } \\
& \begin{aligned}
& d\left[\left(\Pi_{S}^{q}\right)^{*}(C)\right]= \sum_{I}^{\prime} d A_{I} \wedge d X^{I} \\
&= \sum_{I}^{\prime}\left(\sum_{j} \frac{\partial A_{I}}{\partial x^{j}} d x^{j}+\sum_{|\nu| \leq s} \frac{\partial A_{I}}{\partial y_{\nu}} d y_{\nu}\right) \wedge d X^{I} \\
& \equiv \sum_{I}^{\prime} \sum_{j}\left(\frac{\partial A_{I}}{\partial x^{j}}+\sum_{\nu} \frac{\partial A_{I}}{\partial y_{\nu}} y_{\nu+1}\right) d x^{j} \wedge d X^{I} \quad \bmod \Omega_{q} \\
& d C / \Omega_{q}=\sum_{I}^{\prime} \sum_{j}\left(\partial_{j} A_{I}\right) d x^{j} \wedge d X^{I} .
\end{aligned}
\end{aligned}
$$

Hence, $C$ is a conservation law for $R_{q}$ iff

$$
\left(x, y_{\lambda}\right) \in R_{q} \Rightarrow \sum_{I}^{\prime} \sum_{j}\left(\partial_{j} A_{I}\right)\left(x, y_{\mu}\right) d x^{j} \wedge d X^{I}=0,
$$

for all $|\lambda| \leq q,|\mu| \leq s+1$. By exterior algebra, this in fact means

$$
\left(x, y_{\lambda}\right) \in R_{q} \Rightarrow\left[\sum_{j=1}^{k+1} \partial_{i_{j}} A_{i_{1}, \ldots, \hat{i}_{\jmath}, \ldots, i_{k+1}}(-1)^{j-1}\right]\left(x, y_{\mu}\right)=0
$$

for every sequence $i_{1}<i_{2}<\cdots<i_{k+1}$ and where $\hat{i}_{j}$ means that this item is missing in the list.

LeMma 5.1. If $C$ is a conservation law for $R_{p}$, then it is a conservation law for all $R_{t}$ when $t>p$.

Proof. When $p<t<q$, this follows from Definition 5.1. For $t>q$, recall that $\left(\Pi_{q}^{t}\right)\left(R_{t}\right) \subset R_{q}$ by Proposition 3.2, so (5.2) must hold when $\left(x, y_{\lambda}\right) \in R_{t}$.

EXAMPLE 2.1. Replace $(t, x, r, u)$ by $\left(x^{1}, x^{2}, y^{1}, y^{2}\right)$.

$$
R_{1}: y_{10}^{1}+y^{1} y_{01}^{2}+y^{2} y_{01}^{1}=0, \quad C=y^{1} d x^{2}-y^{1} y^{2} d x^{1}
$$

on $J_{0}=X \times Y$, so $s=0$. 


$$
\begin{aligned}
d C= & d y^{1} \wedge d x^{2}-\left(y^{1} d y^{2}+y^{2} d y^{1}\right) \wedge d x^{1} \\
\left(\Pi_{0}^{1}\right)^{*}(d C) \equiv( & \left.y_{10}^{1} d x^{1}+y_{01}^{1} d x^{2}\right) \wedge d x^{1} \\
- & {\left[y^{1}\left(y_{10}^{2} d x^{1}+y_{01}^{2} d x^{2}\right)\right.} \\
& \left.\quad+y^{2}\left(y_{10}^{1} d x^{1}+y_{01}^{1} d x^{2}\right)\right] \wedge d x^{1} \quad \bmod \Omega_{1}, \\
d C / \Omega_{1}= & \left(y_{10}^{1}+y^{1} y_{01}^{2}+y^{2} y_{01}^{1}\right) d x^{1} \wedge d x^{2}=0
\end{aligned}
$$

when $\left(x, y_{\lambda}\right) \in R_{1}$.

EXAMPLE 2.2. Replace $\left(t, x^{1}, y^{1}, \ldots, x^{N}, y^{N}\right)$ by $\left(x, y^{1}, y^{2}, \ldots\right.$, $\left.y^{2 N-1}, y^{2 N}\right)$. Then

$$
C=\sum_{i=1}^{N} m_{i}\left(y^{2 i-1} y_{1}^{2 i}-y^{2 i} y_{1}^{2 i-1}\right)
$$

is a 0 -form (function) on $J_{1}$, so $s=1$. One finds that

$$
d C / \Omega_{2}=\sum m_{i}\left(y^{2 i-1} y_{2}^{2 i}-y^{2 i} y_{2}^{2 i-1}\right) d x^{i}
$$

and, with some algebra, this is zero when $\left(x, y_{\lambda}\right) \in R_{2}$.

To each conservation law $C$ belongs an infinite set of its formal derivatives, each of which is also a conservation law, as we now show.

Definition 5.2. Let $C$ on $J_{s}$ be a conservation law for $R_{p}$ :

$$
C=\sum_{I}^{\prime} A_{I} d X^{I}, \quad A_{I}\left(x, y_{\alpha}\right): J_{s} \rightarrow \mathbb{R} .
$$

Define $\partial_{h} C=\sum_{I}^{\prime}\left(\partial_{h} A_{I}\right) d X^{I}, 1 \leq h \leq n=\operatorname{dim} X$. This is called the formal derivative of $C$ with respect to $x^{h}$. More generally, $\partial_{\nu} C=$ $\sum_{I}^{\prime}\left(\partial_{\nu} A_{I}\right) d X^{I}, \nu=\left(i_{1}, \ldots, i_{n}\right)$.

Note: since $A_{I}: J_{S} \rightarrow \mathbb{R}, \partial_{\nu} A_{I}: J_{S+|\nu|} \rightarrow \mathbb{R}$, so $\partial_{\nu} C$ is a $k$-form on $J_{s+|\nu|} \cdot$

EXAMPLES 2.3, 2.4. Change $(t, x, y)$ to $\left(x^{1}, x^{2}, y\right)$, so

$$
\begin{gathered}
R_{3}: y_{10}-6 y y_{01}+y_{03}=0 . \\
C=y d x^{2}-\left(y_{02}-3 y^{2}\right) d x^{1} \quad \text { on } J_{2} .
\end{gathered}
$$

Instead of $\partial_{x} C$ we have

$$
\begin{aligned}
\partial_{2} C=\partial_{01} C & =y_{01} d x^{2}-\left(y_{03}-6 y y_{01}\right) d x^{1} \text { on } J_{3} . \\
d\left(\partial_{2} C\right) / \Omega_{4} & =\left[y_{11}+y_{04}-6\left(y_{01}\right)^{2}-6 y y_{02}\right] d x^{1} \wedge d x^{2} .
\end{aligned}
$$

But on $R_{4} \partial_{01}\left(y_{10}-6 y y_{01}+y_{03}\right)=y_{11}-6\left(y_{01}\right)^{2}-6 y y_{02}+y_{04}=0$; we conclude that $\left(x, y_{\lambda}\right) \in R_{4}$ implies $d\left(\partial_{2} C\right) / \Omega_{4}\left(x, y_{\lambda}\right)=0$. 
Proposition 5.1. If the $k$-form $C$ is a conservation law for $R_{p}$, then $\partial_{h} C$ is also a conservation law for $R_{p}$.

Proof. Using local coordinates with $C=\sum_{I}^{\prime} A_{I} d X^{I}$, where $A_{I}$ : $J_{s} \rightarrow \mathbb{R}$, we know that for some $q>s$, when $\left(x, y_{\lambda}\right) \in R_{q}$, by (5.3),

$$
\left[\sum_{j=1}^{k+1} \partial_{i_{j}} A_{i_{1}, \ldots, \hat{i}_{j}, \ldots, i_{k+1}}(-1)^{j-1}\right]\left(x, y_{\lambda}\right)=0 \text {. }
$$

Hence by Proposition 3.3, if $\left(x, y_{\lambda}\right) \in R_{q+1}$ then

$$
\begin{aligned}
\partial_{h}\left[\sum_{j=1}^{k+1} \partial_{i_{j}} A_{i_{1}, \ldots, \hat{i}_{j}, \ldots, i_{h+1}}(-1)^{j-1}\right]\left(x, y_{\lambda}\right)=0 \\
\quad=\left[\sum_{j=1}^{k+1} \partial_{i_{j}}\left(\partial_{h} A_{i_{1}, \ldots, \hat{i}_{j}, \ldots, i_{k+1}}(-1)^{j-1}\right]\left(x, y_{\lambda}\right)=0\right.
\end{aligned}
$$

by Proposition 3.1. So, by (5.1) and (5.2) when $\left(x, y_{\lambda}\right) \in R_{q+1}$, $d\left(\partial_{h} C\right) / \Omega_{q+1}=0$.

Definition 5.3. $R_{p}$ has an infinite number of conservation laws if its conservation laws cannot be generated algebraically from laws at some level $J_{p}$ using formal derivatives.

We now prove our main goal, that when $R_{r}^{\prime}$ is a partial prolongation of $R_{p}$, then a conservation law for one of these systems corresponds in a natural way to a conservation law for the other.

Let $R_{p}$ and $R_{r}^{\prime}$ be as in Definition 4.1. Let $C$ on $J_{s}$ be a conservation law for $R_{p}$ so that, for some $q>s, q \geq p$,

$$
C=\sum_{I}^{\prime} A_{I} d X \text { and } d C / \Omega_{q}=\sum_{I}^{\prime} \sum_{i}\left(\partial_{i} A_{i}\right) d x_{i} \wedge d X
$$

and $\left(x, y_{\lambda}\right) \in R_{q}$ implies $d C / \Omega_{q}\left(x, y_{\lambda}\right)=0$.

In fact, of course, $\partial_{i} A_{I}$ depends only on $J_{s+1}=\Pi_{s+1}^{q}\left(J_{q}\right)$. We also have $\Pi_{y}: J_{0}^{\prime}=X \times Y \times Z \rightarrow J_{0}=X \times Y$ and

$$
\rho_{s}\left(\Pi_{y}\right):\left\{\begin{array}{c}
x=x \\
y_{\mu}=y_{\mu}
\end{array}\right\}: J_{s}^{\prime} \rightarrow J_{s} \quad|\mu| \leq s .
$$

Let $P(C)=\rho_{s}\left(\Pi_{y}\right)^{*}(C)=\sum_{I}^{\prime} A_{I} d X^{I}$. In these coordinates, $\Omega_{q}^{\prime}$, the contact form on $J_{q}^{\prime}$, contains all the forms in $\Omega_{q}$, so

$$
d\left[\left(\Pi_{s}^{\prime q}\right)^{*} \circ \rho_{s}\left(\Pi_{y}\right)^{*}(C)\right] \equiv \sum_{I}^{\prime} \sum_{i}\left(\partial_{i} A_{I}\right) d x^{i} \wedge d X^{I} \quad \bmod \Omega_{q}^{\prime},
$$

hence $d(P(C)) / \Omega_{q}^{\prime}=\rho_{q}\left(\Pi_{y}\right)^{*}\left(d C / \Omega_{q}\right)$. 
By condition (3) of Definition 4.1 and Proposition 3.4, $\rho_{q}\left(\Pi_{y}\right)\left(R_{q}^{\prime}\right) \subset$ $R_{q}$, so if $\left(x, y_{\lambda}, z_{\lambda}\right) \in R_{q}^{\prime}$, then $\left(x, y_{\lambda}\right) \in R_{q}$ and since $d P(C) / \Omega_{q}^{\prime}$ and $d C / \Omega_{q}$ have exactly the same expressions which depend only on $\left(x, y_{\lambda}\right),\left(x, y_{\lambda}, z_{\lambda}\right) \in R_{q}^{\prime}$ implies that $d P(C) / \Omega_{q}^{\prime}\left(x, y_{\lambda}, z_{\lambda}\right)=0$. We have proved

Proposition 5.2. If $C$ is a conservation law for $R_{p}$ then $C$ corresponds in a natural way to a conservation law $P(C)$ for $R_{r}^{\prime}$.

Now, let $C^{\prime}$ on $J_{t}^{\prime}$ be a conservation law for $R_{r}^{\prime}$. Suppose that $d C^{\prime} / \Omega_{q}^{\prime}=0$ at points $\left(x, y_{\lambda}, z_{\lambda}\right) \in R_{q}^{\prime}$ for some $q>t, q \geq r$. There is no reason why we cannot suppose $q \geq p$. Let

$$
\begin{aligned}
C^{\prime} & =\sum_{I}^{\prime} B_{I}\left(x, y_{\alpha}, z_{\alpha}\right) d X^{I}, \quad|\alpha| \leq t, \\
d C^{\prime} / \Omega_{q}^{\prime} & =\sum_{I}^{\prime} \sum_{i}\left(\partial_{i} B_{I}\right)\left(x, y_{\beta}, z_{\beta}\right) d x^{i} \wedge d X^{I}, \quad|\beta| \leq t+1,
\end{aligned}
$$

and this is zero whenever $\left(x, y_{\gamma}, z_{\gamma}\right) \in R_{q}^{\prime}$. Using the notations of Definition 4.1,

$$
F:\left\{\begin{array}{l}
x=x, \\
y=y, \\
z=\varphi\left(x, y_{\theta}\right),
\end{array} \quad \rho_{t}(F):\left\{\begin{array}{l}
x=x, \\
y_{\alpha}=y_{\alpha}, \\
z_{\alpha}=\left(\partial_{\alpha} \varphi\right)\left(x, y_{\beta}\right),
\end{array}\right.\right.
$$

where $|\theta| \leq p-r,|\alpha| \leq t$, and $|\beta| \leq p-r+t$, so the "pull-back,"

$$
Q\left(C^{\prime}\right)=\rho_{t}(F)^{*}\left(C^{\prime}\right)=\sum_{I}^{\prime} B_{I}\left(x, y_{\alpha},\left(\partial_{\alpha} \varphi\right)\left(x, y_{\beta}\right)\right) d X^{I}
$$

is a $k$-form on $J_{p-r+t}$.

$d\left[Q\left(C^{\prime}\right)\right]$

$$
\begin{aligned}
& \equiv \sum_{I}^{\prime} \sum_{i} \partial_{i}\left[B_{I}\left(x, y_{\alpha},\left(\partial_{\alpha} \varphi\right)\left(x, y_{\beta}\right)\right)\right] d x^{i} \wedge d X^{I} \bmod \Omega_{p-r+t+1} \\
& =d\left[Q\left(C^{\prime}\right)\right] / \Omega_{q}
\end{aligned}
$$

by definition. But

$$
\begin{aligned}
\partial_{i} B_{I}\left(x, y_{\alpha},\left(\partial_{\alpha} \varphi\right)\left(x, y_{\beta}\right)\right) \\
=\frac{\partial B_{I}}{\partial x^{i}}+\frac{\partial B_{I}}{\partial z_{\alpha}} \frac{\partial\left(\partial_{\alpha} \varphi\right)}{\partial x^{i}}+\frac{\partial B_{I}}{\partial y_{\alpha}} y_{\alpha+1}+\frac{\partial B_{I}}{\partial z_{\alpha}} \frac{\partial\left(\partial_{\alpha} \varphi\right)}{\partial y_{\beta}} y_{\beta+1} \\
=\frac{\partial B_{I}}{\partial x^{i}}+\frac{\partial B_{I}}{\partial y_{\alpha}} y_{\alpha+1}+\frac{\partial B_{I}}{\partial z_{\alpha}}\left[\partial_{i}\left(\partial_{\alpha} \varphi\right)\right] \\
=\left(\partial_{i} B_{I}\right)\left(x, y_{\beta}, \partial_{\beta} \varphi\right), \quad|\beta| \leq p-r+t .
\end{aligned}
$$


Thus, $d\left[Q\left(C^{\prime}\right)\right] / \Omega_{q}=\left(\rho_{t+1}(F)\right)^{*}\left[d C^{\prime} / \Omega_{q}^{\prime}\right]$. If, now, $\left(x, y_{\lambda}\right) \in$ $R_{p-r+q}$, then $\left(x, y_{\lambda},\left(\partial_{\lambda} \varphi\right)\left(x, y_{\beta}\right)\right) \in R_{q}^{\prime}$ by Condition (2) of Definition 4.1 and Proposition 3.4. Since $C^{\prime}$ is a conservation law, when $\left(x, y_{\lambda},\left(\partial_{\lambda} \varphi\right)\left(x, y_{\beta}\right)\right) \in R_{q}^{\prime}, \quad\left(d C^{\prime} / \Omega_{q}^{\prime}\right)\left(x, y_{\lambda},\left(\partial_{\lambda} \varphi\right)\left(x, y_{\beta}\right)\right)=0$.

But, since $d C^{\prime} / \Omega_{q}^{\prime}$ involves only $d x^{1}, \ldots, d x^{n}$,

$$
\begin{aligned}
& \left(d C^{\prime} / \Omega_{q}^{\prime}\right)\left(x, y_{\lambda},\left(\partial_{\lambda} \varphi\right)\left(x, y_{\beta}\right)\right) \\
& \quad=\rho_{t+1}(F)^{*}\left[d C^{\prime} / \Omega_{q}^{\prime}\right]\left(x, y_{\lambda}\right)=d\left[Q\left(C^{\prime}\right)\right] / \Omega_{q} .
\end{aligned}
$$

Hence, the last expression is zero when $\left(x, y_{\lambda}\right) \in R_{p-r+q}$, so $Q\left(C^{\prime}\right)$ is a conservation law for $R_{p}$. We have proved the

Proposition 5.3. If $C^{\prime}$ is a conservation law for $R_{r}^{\prime}$, then $C^{\prime}$ corresponds in a natural way to a conservation law for $R_{p}$.

In order to prove that having an infinite number of conservation laws is shared by systems and their partial prolongations, we must show the correspondences $P$ and $Q$ commute with formal differentiation.

Proposition 5.4. If $C^{\prime}$ on $J_{t}^{\prime}$ is a conservation law for $R_{r}^{\prime}$ then $\partial_{j} Q\left(C^{\prime}\right)=Q\left(\partial_{j} C^{\prime}\right)$.

Proof. Let $C^{\prime}=\sum_{I}^{\prime} B_{I}\left(x, y_{\alpha}, z_{\alpha}\right) d X^{I},|\alpha| \leq t$, so

$$
\begin{aligned}
Q\left(C^{\prime}\right) & =\sum_{I}^{\prime} B_{I}\left(x, y_{\alpha},\left(\partial_{\alpha} \varphi\right)\left(x, y_{\beta}\right)\right) d X^{I}, \quad|\beta| \leq p-r+t, \\
\partial_{j} Q\left(C^{\prime}\right) & =\sum_{I}^{\prime} \partial_{j}\left[B_{I}\left(x, y_{\alpha},\left(\partial_{\alpha} \varphi\right)\left(x, y_{\beta}\right)\right)\right] d X^{I}, \\
& =\sum_{I}^{\prime}\left(\frac{\partial B_{I}}{\partial x^{j}}+\frac{\partial B_{I}}{\partial z_{\alpha}} \frac{\partial\left(\partial_{\alpha} \varphi\right)}{\partial x^{j}}+\frac{\partial B_{I}}{\partial y_{\alpha}} y_{\alpha+1},\right. \\
& \left.+\frac{\partial B_{I}}{\partial z_{\alpha}} \frac{\partial\left(\partial_{\alpha} \varphi\right)}{\partial y_{\beta}} y_{\beta+1}\right) d X^{I} \\
& =\sum_{I}^{\prime}\left(\frac{\partial B_{I}}{\partial x^{j}}+\frac{\partial B_{I}}{\partial y_{\alpha}} y_{\alpha+1,}+\frac{\partial B_{I}}{\partial z_{\alpha}}\left(\partial_{\alpha+1} \varphi\right)\right) d X^{I} \\
& =Q\left(\partial_{j} C^{\prime}\right) .
\end{aligned}
$$

Proposition 5.5. If $C$ on $J_{s}$ is a conservation law for $R_{p}$ then $\partial_{j} P(C)=P\left(\partial_{j} C\right)$. 
The proof is similar to that of Proposition 5.4. Combining these results we have the

THEOREM 5.1. There is a natural correspondence between the conservation laws of a system of pde and one of its partial prolongations. This correspondence commutes with formal differentiation.

In particular, if one system has an infinite number of conservation laws, so does the other.

We now present E. Cartan's response to D. Hilbert's question, when can one say that two systems of pde have a one-to-one correspondence between their solutions?

Definition 5.4 (Recursive). Two systems $R_{p}$ and $R_{r}^{\prime}$ of pde are absolutely equivalent if

(1) $R_{r}^{\prime}$ is a partial prolongation or prolongation of $R_{p}$, or

(2) $R_{p}$ is a partial prolongation or prolongation of $R_{r}^{\prime}$, or

(3) $R_{p}$ is absolutely equivalent to some $R_{s}^{\prime \prime}$ and $R_{s}^{\prime \prime}$ is absolutely equivalent to $R_{r}^{\prime}$.

A property of $R_{p}$ is an absolute invariant when it is shared in a natural way by all systems absolutely equivalent to that system of pde.

We have proved

THEOREM 5.2. The property of having a conservation law is an absolute invariant. The property of having infinitely many conservation laws is an absolute invariant.

\section{REFERENCES}

[1] E. Cartan, Oeuvres Completes, Partie II, Vol 2, Gauthier-Villars, Paris (1953).

[2] P. D. Drazin, Solitons, Cambridge U. Press, Cambridge (1983).

[3] C. Ehresmann, Colloque de Topologie (Espaces Fibres), Masson, Paris (1951).

[4] H. Flanders, Differential Forms, Academic Press (1963).

[5] R. Hermann, Interdisciplinary Mathematics, Vol. XII, The Geometry of NonLinear Differential Equations, Backlund Transformations, and Solitons, Part A, Math. Sci. Press, Brookline, MA (1982).

[6] D. Hilbert, J. Reine Angew. Math., 143 (1913), 300.

[7] H. Johnson, Absolute equivalence of exterior differential forms, Illinois J. Math., 10 (1966), 407-411.

[8] _ The non-invariance of hyperbolicity in partial differential equations, Pacific J. Math., 22 (1967), 419-430.

[9] _ Determination of hyperbolicity by partial prolongations, Pacific J. Math., 30 (1969), 679-695. 
[10] H. Johnson, Partial prolongations and characteristics of differential equations, Trans. Amer. Math. Soc., 146 (1969), 231-240.

[11] _ Conditions for isomorphism in partial differential equations, Pacific J. Math., 39 (1971), 401-406.

[12] P. Lax, Hyperbolic Systems of Conservation Laws and the Mathematical Theory of Shock Waves, Regional Conference Series in Applied Mathematics, vol 11, SIAM, Philadelphia.

[13] F. A. E. Pirani, D. C. Robinson, and W. F. Shadwick, Local Jet Bundle Formulation of Backlund Transformations, Reidel, Dordrecht (1979).

[14] J. F. Pommaret, Systems of Partial Differential Equations and Lie Pseudogroups, Gordon and Breach, New York (1978).

[15] _ Differential Galois Theory, Gordon and Breach, New York (1983).

Received July 25, 1988.

590 S. BRYS

Grosse PoInte Woods, MI 48236 



\section{PACIFIC JOURNAL OF MATHEMATICS EDITORS}

\author{
V. S. VARADARAJAN \\ (Managing Editor) \\ University of California \\ Los Angeles, CA 90024-1555-05 \\ Herbert Clemens \\ University of Utah \\ Salt Lake City, UT 84112 \\ Thomas ENRIGHT \\ University of California, San Diego \\ La Jolla, CA 92093
}

R. FINN

Stanford University

Stanford, CA 94305

Hermann FlaschKa

University of Arizona

Tucson, AZ 85721

VAUGHAN F. R. Jones

University of California

Berkeley, CA 94720

STEVEN KERCKHOFF

Stanford University

Stanford, CA 94305
C. C. MOORE

University of California

Berkeley, CA 94720

Martin SCharlemanN

University of California

Santa Barbara, CA 93106

HAROLd STARK

University of California, San Diego

La Jolla, CA 92093

\section{ASSOCIATE EDITORS}
R. ARENS
E. F. BECKENBACH
B. H. NeumanN
F. WoLF
(1904-1989)
K. YoshidA (1906-1982)

\section{SUP}

UNIVERSITY OF ARIZONA

UNIVERSITY OF BRITISH COLUMBIA

CALIFORNIA INSTITUTE OF TECHNOLOGY

UNIVERSITY OF CALIFORNIA

MONTANA STATE UNIVERSITY

UNIVERSITY OF NEVADA, RENO

NEW MEXICO STATE UNIVERSITY

OREGON STATE UNIVERSITY
UNIVERSITY OF OREGON

UNIVERSITY OF SOUTHERN CALIFORNIA

STANFORD UNIVERSITY

UNIVERSITY OF HAWAII

UNIVERSITY OF TOKYO

UNIVERSITY OF UTAH

WASHINGTON STATE UNIVERSITY

UNIVERSITY OF WASHINGTON 


\section{Pacific Journal of Mathematics}

Vol. 144, No. $1 \quad$ May, 1990

Wojciech Chojnacki, On some totally ergodic functions $\ldots \ldots \ldots \ldots \ldots \ldots 1$

Steven R. Costenoble, Stefan Waner and G. S. Wells, Approximating

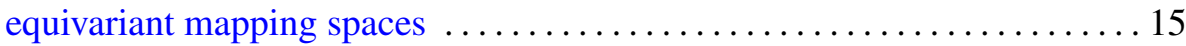

Peter Michael Higgins, A short proof of Isbell's zigzag theorem . . . . . . . 47

Harold H. Johnson, The absolute invariance of conservation laws ........5 51

Edgar Kann, Infinitesimal rigidity of almost-convex oriented polyhedra of arbitrary Euler characteristic $\ldots \ldots \ldots \ldots \ldots \ldots \ldots \ldots \ldots \ldots \ldots \ldots \ldots$

Alan Van Lair, Uniqueness for a nonlinear abstract Cauchy problem . . . . . 105

John B. Little and Kathryn A. Furio, On the distribution of Weierstrass points on irreducible rational nodal curves $\ldots \ldots \ldots \ldots \ldots \ldots \ldots \ldots \ldots 131$

J. S. Okon and Louis Jackson Ratliff, Jr., Reductions of filtrations . . . . . 137

Janusz Pawlikowski, Small subset of the plane which almost contains almost all Borel functions ............................... 155

Sergio A. Tozoni, Vector singular integral operators on a local field ....... 161 John Bason Wagoner, Triangle identities and symmetries of a subshift of

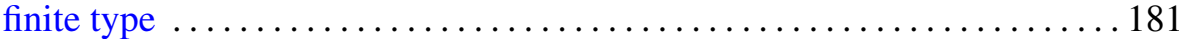

